\title{
A Three-Tier View-Based Methodology for Adapting Human-Agent Collaboration Systems:
}

\author{
Dickson K.W. Chiu ${ }^{1}$, S.C. Cheung ${ }^{2}$, and Ho-fung Leung ${ }^{1}$ \\ ${ }^{1}$ Department of Computer Science and Engineering, The Chinese University of Hong Kong, \\ Shatin, Hong Kong, China. \\ kwchiu@acm.org, lhf@cse.cuhk.edu.hk \\ ${ }^{2}$ Department of Computer Science, Hong Kong University of Science and Technology, \\ Clear Water Bay, Hong Kong, China. \\ scc@es.ust.hk
}

\begin{abstract}
With recent advances in mobile technologies and infrastructures, there are increasing demands for mobile users to connect to existing collaboration systems. This requires extending supports from web browsers on personal computers to SMS, WAP, and PDAs. However, in general, the capabilities and bandwidth of these mobile devices are significantly inferior to desktop computers over wired connections, which have been assumed by most collaboration systems. Instead of redesigning or adapting collaboration systems in an ad-hoc manner for different platforms in a connected society, we propose a methodology of such adaptation based on three tiers of views: user interface views, data views and process views. These views provide customization and help balance security and trust. User interface views provide alternative presentations of inputs and outputs. Data views summarize data over limited bandwidth and display them in different forms. Furthermore, we introduce a novel approach of applying process views to mobile collaboration process adaptation, where mobile users may execute a more concise version or modified procedures. The process view also serves as the centric mechanism for integrating user interface views and data views. This methodology also discusses ways to support external mobile users who have no agent support, customizable degree of agent delegation and the employment of constraint technology for negotiation. We demonstrate the feasibility of our methodology by extending a web-based meeting scheduler into a distributed mobile one.
\end{abstract}

\section{Introduction}

Recent advances in hardware and software technologies have created a plethora of mobile devices [21] with a wide range of communication, computing, and storage capabilities. The Internet is quickly evolving towards a connected society. New mobile applications running on these devices provide users easy access to remote services regardless of where they are, and will soon take advantage of the ubiquity of wireless

\footnotetext{
This work was partially supported by the Hong Kong Research Grant Council with an Ear-
} marked Research Grant (HKUST6187/02E). 
networking to create new virtual worlds. Moreover, as mobile devices become more powerful, peer-to-peer mobile computing will become an increasingly important computation paradigm. These trends in mobile devices and applications have profound implications for application designs in the coming years. However, this connected society will not be a simple add-on to the wired Internet. New challenging problems arise from the handling of mobility, handsets with reduced screens and varying bandwidth. As such, there are increasing demands for the support of collaboration systems across multiple platforms to connect people working together. Research issues for such adaptation involve not only user interface but also collaboration processes as well as enhanced logic within agents.

We have done some work on applying process views in [7] for adapting e-service enactment in a cross-organizational process environment. These views are further refined into an architecture of three tiers, viz., user-interface views, process views, and data views. User interface views provide alternative presentations of inputs and outputs. Data views summarize data over limited bandwidth and display them in different forms. Furthermore, we introduce a novel approach of applying process views to mobile collaboration process adaptation, where mobile users may execute a more concise version or modified procedures. The process view also serves as the centric mechanism for integrating user interface views and data views. Mobile collaboration systems typically involve the support of users with different roles and mobile devices with diverse capabilities. Our views approach presents a flexible mechanism to address this issue. Furthermore, this approach supports both mobile manual human users and different degrees of user delegation to agents in the collaboration system. As such, external users without agent support can also participate in the collaboration system. Our approach can be applied to collaboration systems related to the problems of planning, negotiation and resource allocation. These systems typically involve human users, to be assisted by agents. As an example to demonstrate the feasibility of our approach, we extend an agent-based meeting scheduler to a mobile one.

The contribution and coverage of this paper are as follows: (i) a three-tier viewbased methodology for adapting applications to support mobile platforms, (ii) a novel approach of applying process views in adapting collaboration systems to a mobile environment, (iii) details on the technologies for supporting such adaptations, (iv) support for both human users and user delegated autonomous agents in a collaboration system, (v) use of constraints to limit data exchange in a collaboration system to reduce bandwidth consumption for mobile users, (vi) demonstration of the applicability of our methodology in supporting flexible mobile collaboration systems through these features. The rest of our paper is organized as follows. Section 2 presents the application requirement of the distributed mobile meeting scheduler with respect to mobile platforms, together with related work. Section 3 summarizes our three-tier view-based framework and architecture for mobile collaboration, supporting both manual human users and agents. Section 4, 5, and 6 discusses customization through these views one by one. Section 7 presents the internal architecture of these distributed mobile agents and how we adapt them. We conclude this paper with our plans for further research in Section 8. 


\section{Motivating Example and Related Work}

Meeting scheduling is a common collaboration task that is time-consuming and tedious. It involves negotiation between two persons or among several persons, taking into account many factors or constraints. In our daily life, meeting scheduling is often performed by ourselves or by our secretaries via telephone or e-mail. Most of the time, each attendee has some uncertain and incomplete knowledge about the preferences and calendar of the other attendees. Thus a meeting scheduler is a very useful tool for group collaborations. Meeting scheduling is one of the classic problems in artificial intelligence and multi-agent systems. There are some commercial products but they are just calendars with special features, such as availability checkers, meeting reminders [15. Shitani [31] highlighted a negotiation approach among agents for a distributed meeting scheduler based on the multi-attribute utility theory. Van Lamsweerde [22121] discussed goal-directed elaboration of requirements for a meeting scheduler, but did not discuss any implementation frameworks. Sandip 30] summarized an agent-based system for automated distribution meeting scheduler, but was not based on BDI agent architecture. However, these systems cannot support manual interactions in the decision process or any mobile support issues. Therefore we use this application as an example for our methodology.

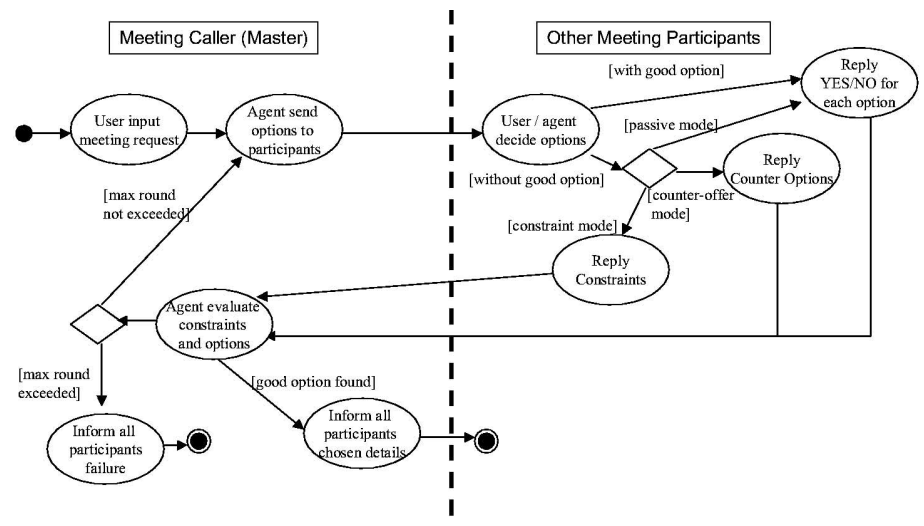

Fig. 1. Activity Diagram for Agent-based Meeting Scheduler

Fig. 1 depicts the activity diagram of an agent-based meeting scheduler. The user who proposes a meeting (or meeting proposer) enters his/her requirements and suggestions (or options) and delegates the agent to schedule the meeting. The delegated agent then contacts the other meeting participants or their delegated meeting scheduler. Each of the participants or agents then determines if any options are good. If so, the decision can simply be passed back to the proposer's agent. Otherwise, depending on the platform (cf. Fig. 2), a participant might reply in the following three respond modes: (i) Passive mode - the participant (or his agent) just reply that all the proposed schedules are bad, without any counter proposals. (ii) Counter-offer mode - the participant (or his agent) suggests other counter proposals. (iii) Constraint mode - the participant (or his agent) gives some of the constraints representing the participant's 
preference and availability. After gathering the feedback, the proposer's agent evaluates it for a common feasible solution for all participants. If this is successful, all participants will be informed of the result. Otherwise, the proposer's agent will attempt another round of proposals, with the consideration of the options already rejected by the participants and their constraints (if any). However, if the maximum round is exceeded, the proposer's agent will consider the scheduling failed and inform all participants.

\begin{tabular}{|l|l|l|l|}
\hline \multicolumn{1}{|c|}{ Platforms } & PDA & WAP & SMS \\
\hline $\begin{array}{l}\text { Meatures } \\
\text { requestor }\end{array}$ & Program interface & WAP interface & SMS \\
\hline $\begin{array}{l}\text { Agent } \\
\text { delegation }\end{array}$ & Agent run on PDA & Agent run on server \\
\hline Alerts & $\begin{array}{l}\text { ICQ, email (or SMS if } \\
\text { user also accessible) }\end{array}$ & SMS & WAP interface \\
\hline $\begin{array}{l}\text { Exception } \\
\text { handling or } \\
\text { manual mode } \\
\text { for participants }\end{array}$ & Web interface & SMS \\
\hline $\begin{array}{l}\text { Participant } \\
\text { response mode }\end{array}$ & $\begin{array}{l}\text { Passive, Counter-offer, } \\
\text { Constraint }\end{array}$ & $\begin{array}{l}\text { Passive, Counter- } \\
\text { offer }\end{array}$ & Passive \\
\hline
\end{tabular}

Fig. 2. Different Features for Users of Meeting Scheduler on Different Platforms

This approach is quite ad-hoc and cannot describe customization in other activities of this process in an orderly manner. In case we model a mobile e-service process using the existing process definition infrastructure, we can use branches to represent existing alternatives because of the different platforms that a user might use. If the representation ends there, then we are unable to differentiate between branches that represent alternative execution paths from those represent different actions due to the use of mobile devices. In the later case, the designer has to specify the tasks to be executed, based on the constraints imposed by the platform and also has to associate the corresponding data that restrict the information to be displayed and to specify the interface that defines how to present the data based on the capabilities of the displaying device. Therefore, we need to augment the specification process such that the designer can customize the process definition in an orderly and consistent way taking into account all the above aspects. We propose to address this problem using views.

Our ideas in process views for cross-organizational business interaction and econtract enactment have been presented in 7]. This approach has been motivated by views in object-oriented data models, which can be dated back to [11], and in particular by imaginary objects in [1], which introduced the concept of inheritance of a public process from a private process to achieve interoperability in a cross-organizational ecommerce environment. Gardarin [14] discussed federated OODBMS and views for objects in a distributed environment. Liu and Shen [24 presented an algorithm for process view construction and verification, but did not discuss any of its applications. Most ongoing efforts are however target at user interface views only, such as the W3C initiative on device independence [35], Oracle's Wireless Edition [29], and IBM's Websphere Transcoding Publisher [17]. 
Advanced workflow management systems are now web-enabled, but only very recently researchers in process technologies are exploring mobile process support. The WHAM (process enhancement for mobility) prototype [19] supports mobile workforce and applications in a process environment, with a focus on network connectivity and the mobility of process resources. Tjao [34] introduced a Java Border Service Architecture, which is an abstract layer between presentation and application logic of an application, to handle mainly user interface issues of mobile devices, using process as an example. As for commercial products, Staffware [32] has recently introduced, WAP Business Process Server. However, all of them do not support platform specific process adaptation or integrated platform independent solution. Neither do they support view mechanisms. We are now extending our web-enabled WFMS, E-ADOME [7][8][9], to ME-ADOME [5] in order to support mobile users effectively (via SMS, WAP, and PDA browsers) with a three-tier view-based mechanism.

In summary, the three-tier view approach presented in this paper is a novel approach to adapting mobile collaboration systems. Other meeting scheduling systems cannot support manual interactions in the decision process or does not adequately address mobile support issues. Comparing with the systems close to us, our meeting scheduling system has the most features and can support both mobile agents and manual human users on the Internet.

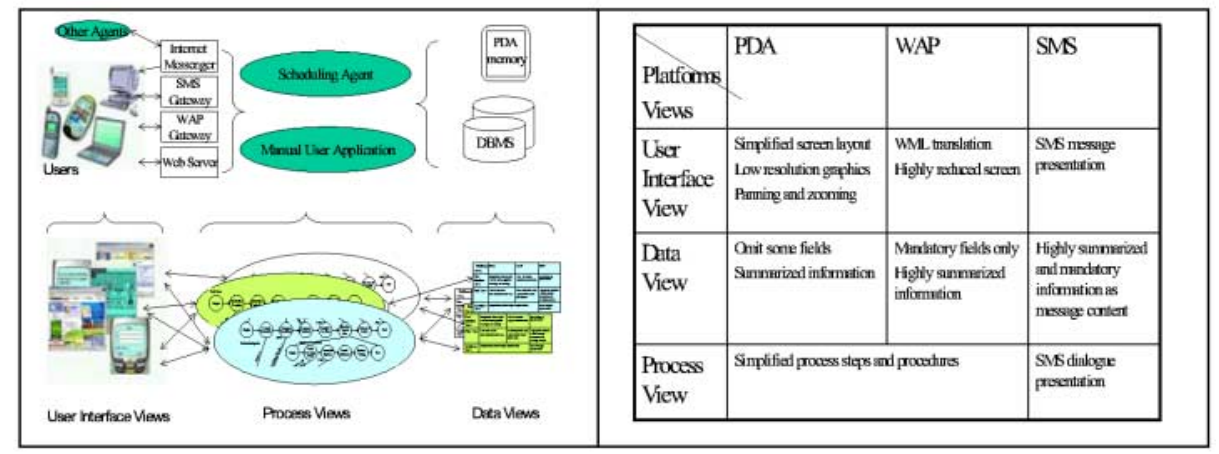

Fig. 3. Three-Tier Architecture and Three-Tier Views

\section{Multiple-Platform Adaptation with a Three-Tier View-Based Methodology}

Internet applications are generally developed with a three-tier architecture comprising front ends, application servers and back end databases. Each of these tiers hosts a set of views as shown in $\mathbf{F}$ g. 3, which also depicts our implementation architecture. It also summarizes the contribution of different types of views to users on these mobile platforms respectively, addressing the technological limitations of different mobile platforms, viz. PDA, WAP and SMS, with reference to mobile E-service requirements.

Views help balance trust and security, that is, only information necessary for the process enactment, enforcement and monitoring of the contract is made available to the concerned parties, in a fully control and understandable manner. At the front-end 
tier, user interface views provide mobile users with alternative presentations and interfaces to interact with the process views hosted at the application servers tier. User interface views can be developed based on the technology of XML Style Language (XSL) [36]. A user interface view consists of multiple screens and forms based on the capabilities of the front end devices. For example, Fig. 9(a) and (b) give the screens in two user interface views for devices with and wi hout graphical capabilities, respectively. A process view is a projection of an E-service process that concerns a user and his/her front-end device. Process views are synchronized and enacted by application servers. A data view consists of multiple tables that collectively represent a projection of data that are required in the enactment of an E-service.

We separate user alerts from user sessions to improve the flexibility [9]. Online users will be alerted by ICQ [18], with the task summary and reply URL as the message content. If a user is not onlne or does not reply within a pre-defined period, the application server will send the alert by email. At the same time, another alert may be sent via SMS to the user's mobile phone. Whatever the alert channel has been, the user need not connect to application server on the same device, or even on the same platform. For example, after receiving a SMS alert, the user may use a handset to connect to the application server via WAP, or reply with an SMS message. Alternatively, the user may find a PC with Internet connection or use a PDA to connect to the application server.

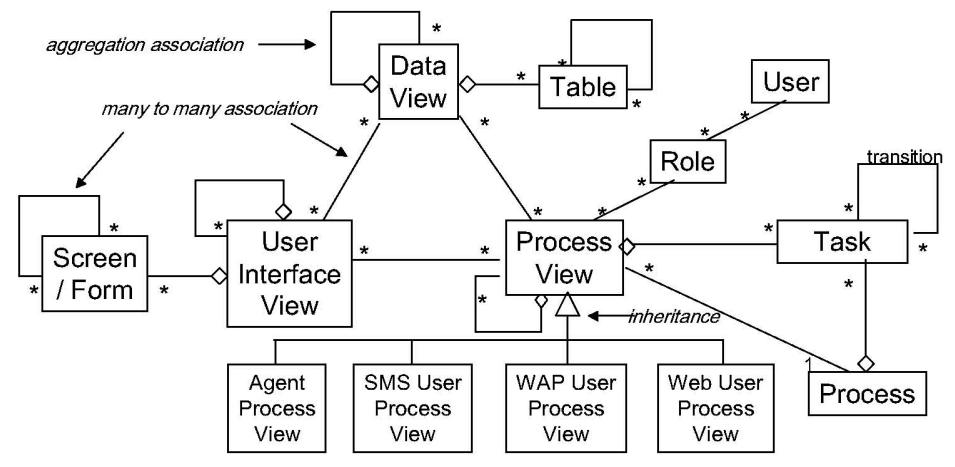

Fig. 4. Meta-model of Three-tier Views in UML Class Diagram

Fig. 4 presents our meta-model of the three-tier views. User interface, process and da a views are related by many-to-many relations. In other words, a user interface view may provide the interface to multiple process views, each of which may in turn supports multiple user interface views. The scenario of extending an existing webbased application to support mobile users is usually more typical than designing a multi-platform application from scratch. In addition, the following discussion is also applicable to phased projects in which usually standard web-based application are developed as the first phase, and supporting mobile users as subsequent phases. We propose the design of the views in three tiers to be carried out in the following major steps: 
1. Consider the new use cases (corresponding to the new platforms) to be added and their requirements.

2. For each new user case, consider the impact of these new requirements on existing processes. Do we need to adapt the processes? Design process views accordingly.

3. Design data views based on the requirement of the process views.

4. Design user interface views based on platform dependent restrictions.

5. Update the logic in the agents to accommodate the above changes.

\section{Adaptation with Process Views}

Motivated by views in federated object databases [14], we propose the use of process views as a fundamental mechanism for flexible collaboration adaptation. A process view is a structurally correct subset of a process definition (as defined in [7], [16]). We develop integrity criteria concerning the consistency between workflow views and their parent workflows, and correctness criteria concerning the consistency between workflow views and their target communication scenarios [6]. In this paper, we further propose to use the concept of process views to let different users (on different platforms) access different customized version of the same process. Within an organization, process views are also useful for security applications, such as to restrict accesses (like the use of views in databases). Thus, process views serve as the centric mechanism in our approach, i.e., process views represent customized business processes that integrate with data views and user interface views, as illustrated with our motivating example in the previous section. Based on our meta-model presented in Fig. 4, the components of a process view include the customized process flow graph, access control, related data views and user interface views:

Access (Security) Control - Each process view must be specified with one or more accessible roles with an access statement. A role represents a collection of users of similar properties [8]. While some roles (e.g., SMS, WAP, Web) are used to distinguish user platforms for mobile process support, other roles (e.g. director, manager, officer) may also be used in specifying security context.

Process Flow Graph - Most contemporary WFMSs use a hierarchical composition approach, i.e., a process is composed of sub-processes and so on down to leaf-nodes of atomic activities. This provides a good granularity for providing views of the process flow graph. If a process view is to be made available, a fundamental provision is the topmost level process flow graph. However, the detailed composition of individual sub-process may be concealed. Thus a process in the flow graph can be presented in one of the following ways: (i) a white-box sub-process is specified with a sub-process view by a statement "process p1 view v1" (i.e., the details of the sub-process is further visible and subject to the restriction of a sub-process view); (ii) a black-box subprocess (is limited from further details of its further internal composition 1 , (iii) a gray box where some sub-processes are visible while other sub-processes are concealed. Similarly, we can define customized parts the process flow graph for different parts of

${ }^{1}$ Unless a view is specified for the sub-process, it is a black box. 
a composite process. Transitions among processes are specified with the transition statement with optional guard conditions.

Data views associated with a process instance - Data tables associated with a process need not be presented completely in a process view. Some fields may be hidden from the views, some may be read only, while some may be presented with write access. Data views are specified with the dataview statement. We omit further details of data views as they can be specified with standard Structure Query Language (SQL) statements 12. Further examples are given in Section 5.

User interface views associated with a process instance - User interface views (further specified in XSL) associated with a process specify different user interfaces for users on different platforms. Further details and examples are given in the next sub-section.

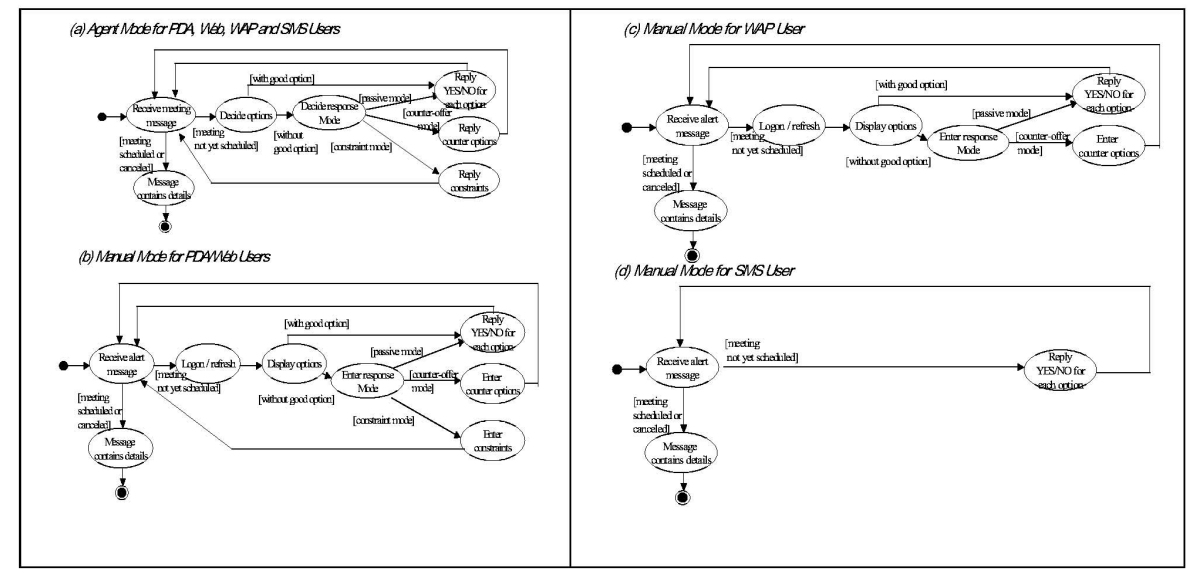

Fig. 5. Different Process Views for Participants on Different Platforms

Fig. 5 summarizes the details of the process views that take place at the meeting participants other than the meeting proposer, i.e., these process views address the differences among the agent-delegated process (Fig. 5a) and customized processes for users on different platforms (Fig. 5b-d). After receiving an alert via ICQ or email, a PDA user (or other users on a web browser) or WAP user in manual mode log on the manual user application on a web server to review the detailed information of the meeting proposal. In addition, the user may also access his timetable from the application or as a separate task (e.g., a separate program in his PDA or phone) whichever appropriate. The user then determines if any options are good. If so, the decision can be passed back to the proposer's agent through the web or WAP interface respectively. Otherwise, a PDA user might reply in any of these three respond mode described in the previous subsection. However, the constraint mode is too complicated to be supported through a WAP interface and is therefore not provided to WAP users. As for a SMS user, the only practical option is to reply with a SMS message, as other ways of responding is far too complicated. 
In general, there is no universal recipe for designing process views, as this process is analogous to designing a piece of customized software. In order to capture the requirements for users of a mobile platform, a commonly adopted approach from objectoriented design is to carry out use case analysis. We should concentrate on the difference of the requirements for the mobile users from those under standard browsers. These differences are compared with the standard processes to formulate views. We should identify similar or identical tasks to maximize reuse, and in particular, consider the possibility of customizing them with data views and user interface views instead of rewriting them.

Usually, a complete detailed business process is too complicated for a mobile environment. Therefore, typical requirements are simplification of the process, reordering of work steps, delegation of tasks (work steps) to other personal, etc. For example, a user with only SMS support can only make very simple decisions or feedback. As this kind of operating environment is often error prone and may have security problems, we suggest not allowing critical options in these process views. On the other hand, it is often difficult to tell if a user can tolerate a complex process because of the operating environment or even due to the user's mood, which cannot be determined merely with observation facts, such as the mobile platform and physical location. Therefore, it is always a good idea to allow users to choose their desired view options whenever feasible.

Mobile applications may be enhanced by using the awareness of the user's location, and location dependent information available and relevant to the application, e.g., mobile workforce management systems and provision of information to mobile users. Exception handling due to unavailability of the required personnel, network delay, interrupts and disconnections, etc., should also be considered. In the worst case of aborting a process, we should consider additional requirements for compensation processes (e.g., an order cancellation) under a mobile environment.

\section{Adaptation with Data Views}

A data view is a set of tables comprising a projection of the enterprise data that are required for the enactment of some process views. Fig. 6 presents visually the schema of a data view in UML Class Diagram for the Display options process view. Note that a class or an association in the diagram maps to a data table with columns representing the class attributes [4]. The main table in this data view is the Proposal table, which consists of a number of proposals made by different proposers. A proposal can be a counter-offer of a previous proposal. Each proposal contains a description and the time when the proposal was made. A proposal consists of constraints in venue and time. Multiple available time slots can be suggested in an option.

The data view for Display option process view is a projection and selection of the entire data set for the meeting scheduler. Fig. 7 presents the entire schema for the mobile meeting scheduler example. As shown in the figure, the data view in Fig. 6 is a selected subset of data objects as well as a projection of some data object fields. 


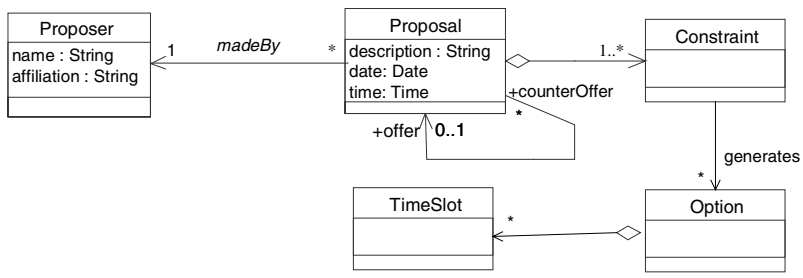

Fig. 6. The UML Schema of a Data View for WAP Manual Mode Process View

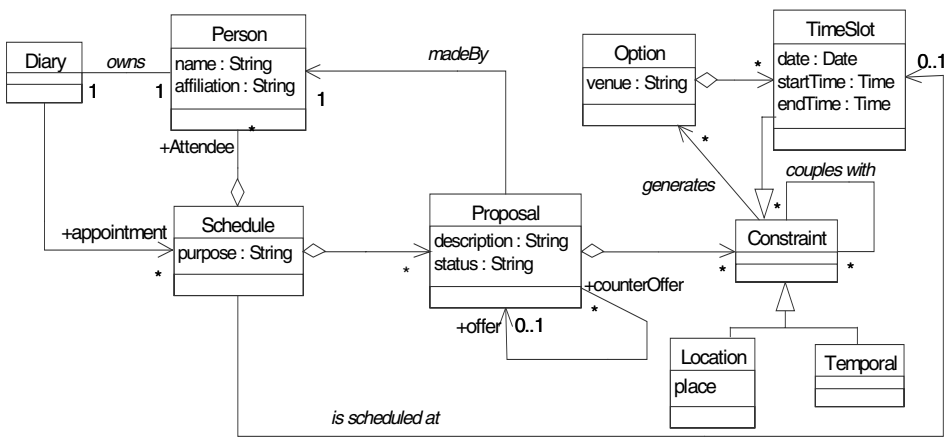

Fig. 7. The UML Schema for the Mobile Meeting Scheduler System

With a process view defined, we can proceed to analyze its data requirement. Each work step requires data from the database in some form. In particular, we should identify mandatory fields, optional fields and fields that are to be skipped in the view, in order to cope with the simplification required for mobile users. However, additional fields those have to be computed for summarizing information and knowledge may be required. In case that the mobile user cannot provide mandatory information or input them effectively, we may need to modify the process so that the mandatory information can be provided later or by someone else. Therefore, like designing software, the process is not a linear one. In addition, security requirements should also be considered, e.g., sensitive information may have to be restricted to users within the office or to those of pre-approved locations. Data views may also be employed to hide less important data field or to show alternate summary columns.

\section{Adaptation with User Interface Views}

User interface views provide users with appropriate interfaces to interact with process views within the capabilities of front end devices. This means the user interface views for a web user can be different from that for a WAP user. Fig. 8 presents two possible user interface views that support the Display options task in the process views depicted in Fig. 5 for web users and WAP users, respectively. Both Fig 8(a) and (b) cor- 
respond to the same XML document object, but are rendered by two different XSL style sheets.

(a)

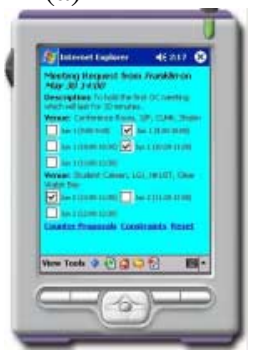

(b)

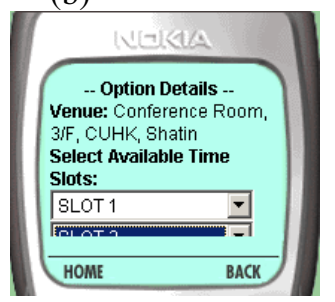

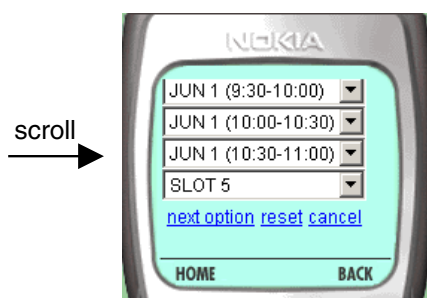

Fig. 8. Two Types of Display Option Screens for (a) PDA and (b) WAP Users

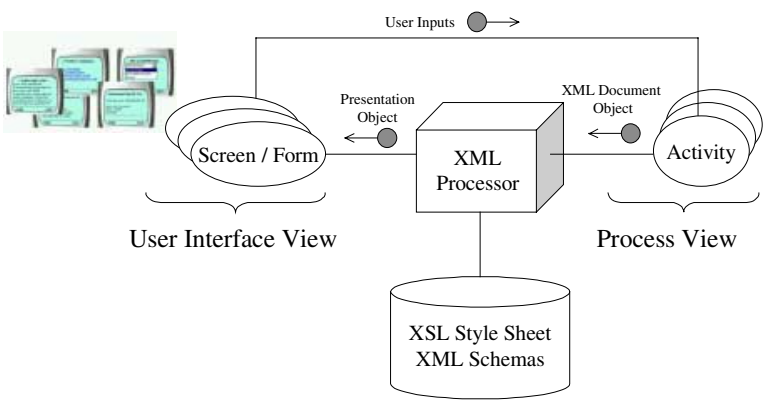

Fig. 9. Implementation Framework for User Interface Views

The differences between the process views for the PDA user and those for the WAP user are minor. However, these two processes need to be customized at the user interface tier instead, i.e., the appearance of the screens and input is different while they are referring to the same set of information items. User interface views can be facilitated by contemporary XML technologies augmented with XSL in elegantly, as further explained in the next section. Similarly, users on PDAs and PCs may also prefer different user interface views to cater for the difference in screen size.

Fig. 9 presents the implementation framework for user interface views using XSL technology [36]. Information to be presented at a user interface view is structured as XML document objects by a process view, such as the WAP based process view in Fig. 5(b). For example, an XML document object of a meeting proposal is generated by the Display Option activity. XML document objects are then transformed by an XML processor into presentation objects based on specified XML schemas and XSL style sheets. The presentation objects for a WAP user interface view are decks and cards in the Wireless Markup Language (WML). 
We usually need to remove graphics or reduce the resolution, provide panning and zooming, shorten fields or provide summarized ones instead, break one web page into several screens, etc. For user input, we should consider the difficulties in entering data (especially typing) on mobile devices, and provide menu selections as far as possible. For PDA interface, the main problem is just a smaller screen, some of which may be black-and-white. If the original full-function user interface is too complicated (e.g., too many unnecessary features or high resolution screen layout), another simplified user interface view is probably required. Pictures and documents may require to be shown in lower resolution and documents may be outlined and level-structured. Panning and zooming (supported by most browsers) should also help. For users on a WAP interface connecting to the application server via a WAP gateway, the screen is extremely small. A user interface view is mandatory to map the original one into WML.

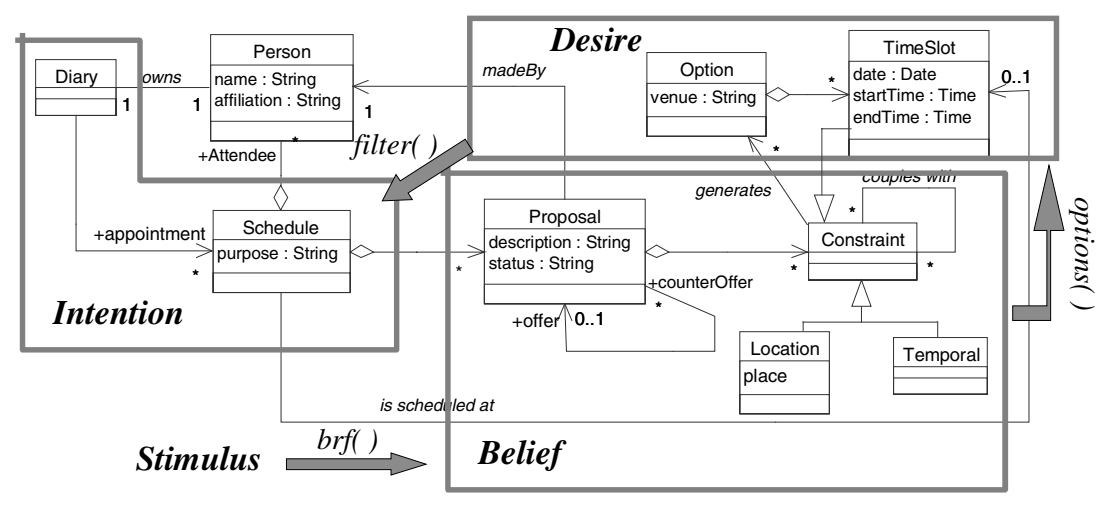

Fig. 10. Mapping Between Data Schema in Fig. 7 and BDI Architecture

\section{Adapting the Decision Agent}

After presenting our three-tier view-based approach and the application environment, we proceed to discuss the architecture of the agents for human-agent collaboration and how we adapt them to a mobile environment in this section. Fig. 10 summarizes the implementation of the data schema using Belief-Desire-Intention (BDI) agent architecture. Our BDI architecture is composed of three main data sets: belief, desire, and intention. Information or data are passed from one data set to another through the application of some functions. Once the percepts are sensed as input, the belief revision function ( $b r f$ ) converts it to a belief. The desire set is updated by generating some options based on the data in belief set. Options in desire set are then filtered to new intentions of the agent, and a corresponding action is then outputted. In our meeting scheduler, a BDI agent simulates a secretary for arranging appointments on behalf of a human user. Thick arrows indicate update operations. Here, we have not explicitly elaborated the plan in the mapping because it is outside the scope of the scheduler. For 
instance, the plan to attend a meeting may consist of taking a bus and then change for a train. In general, a plan of an agent involves proper resource allocation so as to realize its intentions.

Constraint satisfaction [25] [33] [26] is one of the major research areas in Artificial Intelligence. Many real life problems, which cannot be easily modeled in specific mathematical forms and solved by conventional Operations Research methods, may be expressed as constraint satisfaction problems. In its basic form, a constraint satisfaction problem consists of a finite number of variables, each ranging over its own finite domain of discrete values, and a finite number of constraints. Each constraint is a relation over a subset of the variables, restricting the combinations of values these variables that they can take. Formally, a Constraint Satisfaction Problem is a tuple $<X$, $D, C>$, where $X$ is a finite set of variables, $D$ a function mapping a variable to its domain, and $C$ a finite set of constraints. For any variable $x$ in $X$, we require that $D(x)$ is a finite set of discrete constants. A constraint $c\left(y_{1}, y_{2}, \ldots, y_{n}\right)$ in $C$ is a relation over a finite subset $\left\{y_{1}, y_{2}, \ldots, y_{n}\right\}$ of $n$ variables in $X$. A solution to a constraint satisfaction problem is an assignment of values to variables so that all constraints are satisfied. In a meeting-scheduling problem, the two main issues to be determined are the time and place of a meeting. Usually, a user has a number of possible options for the time and place of a meeting. Each of these options is usually associated with a degree of preference so that the feasibility of these options can be interrelated.

Once all the users requirements are represented as constraints, conventional constraint solving method, such as systematic search [21], possibly enhanced by constraint propagation [3] 21] [27], can be applied to find the feasible day, time and place for a particular meeting. However, since all these conventional solvers are centralized, it is inappropriate to employ any of them in a multi-agent mobile setting. This is because employing a centralized solver implies having to require every involved agent to send all or part of personal calendars to a designated agent, which is supposed to find out a feasible solution to the meeting-scheduling problem. Obviously, this is inappropriate in multi-agent systems, in which all agents are supposed to enjoy privacy protection and autonomy. Moreover, there is no way to avoid the designated agent's selfish yet rational behavior of arranging the meeting at a day, time and place that is most convenient to itself only.

We observe that any meeting scheduling protocol involves a trade-off between privacy protection, message exchange costs, and computational efficiency of scheduling. The protocol we describe in the previous paragraph, which commonly known as opencalendar protocol is computational efficient yet provides no privacy protection. Another problem is that too much unnecessary data is sent. Therefore this approach wastes bandwidth and is not suitable for mobile users or agents. On the other extreme, the most privacy-protected protocol is to require, say, Mary, to make a sequence of specific suggestions to Franklin. Each of these suggestions consists of a specific day, a specific time on that day, and a specific place. Franklin considers each of them and decides whether or not to accept the suggestion. This protocol, which we call the passive mode, is a simple, inefficient protocol (may be causing too many exchanges of short messages) that provides high degree of privacy protection. We can see that there is a spectrum of protocols in between these two extremes, which require the agents to 
exchange their private information to a certain degree. For example, in the counteroffer mode implemented, the meeting proposing agent first sends several options to other participants, who are expected to indicate the feasibility of each of these individual options. Each participant may also counter-propose by replying with a set of his/her own constraints. As such, a balance among privacy protection, message exchange costs, and computational efficiency can be achieved.

Another issue concerning meeting scheduling is the recognition of users' preferences. In our implementation, user preferences are recognized by associating a solution evaluation function defined according to the user's preferences, and enhance the tree search to a branch-and-bound search strategy [21]. In adaptation towards mobility, the agent should be aware of the current locations of all the participants, i.e., the stimulus should include this information. This information is used for ensuring that the participants can arrive at the meeting place on time. We also need to extend the belief, intention, and desire data sets to accommodate for this information, as well as to extend the brf, options, and filter functions accordingly.

Our origin project aimed at exploring the feasibility of implementing agents on PDA platforms. We implemented them in Microsoft embedded Visual C++. We found that the mobile agents can run a good speed on a PDA over a wireless local area network (LAN), i.e., they can solve the constraints in a few seconds. As such, this distributed architecture does not need a centralized server and therefore can be highly scalable, especially noting that there may be many concurrent negotiations but the participants involving in a single negotiation are usually few.

\section{Conclusions and Further Work}

This paper has presented a pragmatic three-tier view-based methodology in adapting various types of users and agents, in particular wireless mobile ones, in a connected society for collaboration systems. This paper has also introduced a three-tier viewbased architecture (viz., process view, user interface view and data view) to provide such support. We have demonstrated the feasibility of our methodology by showing how a meeting scheduler can be adapted to accomplish such objectives. Compared with other researches on this topic, our methodology employs an improved environment through standard state-of-the-art technologies, which can adapt to changing requirements of mobile users and devices, with extensive support for reuse. Further note that, specification of process views is based on standard UML activity diagram, many of the techniques presented in this paper can thus be applicable to other systems. In addition, the concepts of user interface view and data view should be widely applicable to a wide range of mobile applications.

In our current project, we aim at extending the system to support mobile users in a connected society. Currently, we implement the agents on PC-based server platforms in Java as well. We further include location information to these scheduling agents. On the other hand, we implement the applications to support manual user decisions with our three-tier view methodology. The next phase of our project is to port the Java 
agents from PC platforms to PDAs by using J2EE to test whether newer PDAs can support agent implementation effectively when we can acquire them later.

For process views, we are working on further details of formal definitions, construction and verification algorithms, more detailed taxonomy, view update mechanisms, and more operations support. In addition, we are interested in the application of our three-tier view-based approach in various advanced real-life e-commerce environments, such as procurement, finance, stock trading and insurance. In particular, we are looking into location depend application, such as mobile workforce management and mobile customer relationship management (CRM) applications. For agent-based application, we are investigating exceptions and their automatic handling, such as cancellations and rescheduling.

\section{References}

1. S. Abiteboul and A. Bonner. Objects and Views. In Proceedings of ACM SIGMOD Conference, pp. 238-247, 1991.

2. G. Alonso, et al. Exotica/FMDC: a Process Management System for Mobile and Disconnected clients. Distributed \& Parallel Databases, 4(3):229-247, 1996.

3. Bessière, C. and Cordier, M. Arc-consistency and Arc-consistency again. In Proceedings AAAI-93, pp. 108-113, 1993.

4. D. Carlson. Modeling XML Applications with UML, Addison-Wesley, 2001.

5. D.K.W. Chiu, S.C. Cheung and E. Kafeza. View-based Support for Mobile Workflow. In Proceedings of 1 st International Conference on Mobile Business, CDROM, Athens, Greece, July 2002.

6. D.K.W. Chiu, S.C. Cheung, Sven Till, K. Karlapalem, Q. Li, and E. Kafeza. Workflow View Driven Cross-Organizational Interoperability in a Web Service Environment. Information Technology and Management, Kluwer Academic Publishers, 2003 (to appear).

7. D.K.W. Chiu, K. Karlapalem, Q. Li and E. Kafeza. Process Views Based E-Contracts in a Cross-Organization E-Service Environment. Distributed and Parallel Databases, Kluwer Academic Publishers, 12(2-3):193-216, 2002.

8. D.K.W. Chiu, Q. Li and K. Karlapalem. A Meta Modeling Approach for Process Management System Supporting Exception Handling. Information Systems, 24(2):159-184, 1999.

9. D.K.W. Chiu, Q. Li and K. Karlapalem. Web Interface-Driven Cooperative Exception Handling in ADOME Process Management System. Information Systems, Pergamon Press, Elservier Science, 26(2):93-120, 2001.

10. V. Chopra, et. al. Professional XML Web Services. Wrox Press, 2001.

11. U. Dayal. Queries and Views in an Object-Oriented Data Model. In Proceedings $2^{\text {nd }}$ International Workshop on Database Programming Languages, 1989.

12. R. A. Elmasri and S. B. Navathe. Fundamentals of Database Systems. Addison-Wesley, 3rd edition, 2000.

13. H. Evans and P. Ashworth. Getting Started with WAP and WML. Sybex, 2001.

14. G. Gardarin, B. Finance and P. Fankhauser. Federating object-oriented and relational databases: the IRO-DB experience. In Proceedings of the 2nd IFCIS International Conference on Cooperative Information Systems (CoopIS '97), pp. 2-13, 1997. 
15. L. Garrido, R. Brena and K. Sycara. Cognitive Modeling and Group Adaptation in Intelligent Multi-Agent Meeting Scheduling. In Proceedings of First Iberoamerican Workshop on Distributed Artificial Intelligence and Multi-Agent Systems, pp. 55-72, 1996.

16. A. ter Hofstede, M. Orlowska and J. Rajapakse. Verification Problems in Conceptual Process Specifications. Data \& Knowledge Engineering, Pergamon Press, Elservier Science, 24(3) 239-256, 1998.

17. IBM Redbooks. New Capabilities in IBM Websphere Transcoding Publisher Version 3.5 Extending Web Applications to the Pervasive World. IBM Corp., 2001.

18. ICQ. http://www.icq.com

19. J. Jing, K. Huff, B. Hurwitz, H. Sinha, B. Robinson and M. Feblowitz. WHAM: Supporting Mobile Workforce and Applications in Process Environments. In Proceedings of 10th International Workshop on Research Issues in Data Engineering, San Diego, California, IEEE Press, pp. 31-38, 2000.

20. G. Kappel, B. Pröll, W. Retschitzegger and W. Schwinger. Customization for Ubiquitous Web Applications - A Comparison of Approaches. Int. Journal of Web Engineering and Technology (IJWET), Inaugural Volume, Inderscience Publishers 2003.

21. V. Kumar. Algorithms for Constraint-Satisfaction Problems: A Survey. AI Magazine, 13(1)32-44, 1998.

22. A. van Lamsweerde, R. Darimont and P. Massonet. Goal-Directed Elaboration of Requirements for a Meeting Scheduler: Problems and Lessons Learnt. In Proceedings of Second IEEE International Symposium on Requirements Engineering (RE '95), pp. 194-203, 1995.

23. Y.-B. Lin and I. Chlamtac. Wireless and Mobile Network Architectures. John Wiley \& Sons, 2000.

24. D.-R. Liu and M. Shen. Modeling Processs with a Process-View Approach. In Proceedings of 7th International Conference on Database Systems for Advanced Applications (DASFAA 2001), April 2001, Hong Kong, IEEE Computer Society, pp. 260-267

25. Mackworth, A. K. Consistency in Networks of Relations. Artificial Intelligence, 8(1) 99$118,1977$.

26. Marriott, K. and Stuckey, P. J. Programming with Constraints: an Introduction. The MIT Press, 1998.

27. Mohr, R. and Henderson, T. C. Arc and Path Consistency Revisited. Artificial Intelligence, 28:225-233, 1986.

28. Object Management Group. Foreword UML specification 1.4, September 2001.

29. Oracle Inc. Oracle9iAS Wireless. http://www.oracle.com/ip/deploy/ias/index.html?wireless.html

30. Sandip, S. Developing an Automated Distributed Meeting Scheduler. IEEE Expert, pp. 41-45, 7/8-1997.

31. T. Shitani, T. Ito and K. Sycara. Multiple Negotiations among Agents for a Distributed Meeting Scheduler. In Proceedings of $4^{\text {th }}$ International Conference on MultiAgent Systems, July, 2000, pp. 435-436.

32. Staffware Corporation. Staffware Global - Staffware's Opportunity to Dominate Intranet based Process Automation, 2000, http://www.staffware.com

33. Tsang, E. Foundations of Constraint Satisfaction. Academic Press, 1993.

34. A. M. Tjoa, R. R. Wagner and A. Al-Zobaidie. On Integrating Mobile Devices into a Process Management Scenario. In Proceedings of 11th International Workshop on Database and Expert Systems Applications (DEXA 2000), IEEE Press, pp. 186-192, 2000.

35. W3C. Device Independence Activity. http://www.w3.org/2001/di/

36. http://www.w3.org/XML/ 